\title{
The precision of spatial selection into the focus of attention in working memory
}

\author{
Alessandra S. Souza $^{1} \cdot$ Mirko Thalmann $^{1} \cdot$ Klaus Oberauer $^{1}$
}

Published online: 23 April 2018

(C) Psychonomic Society, Inc. 2018

\begin{abstract}
Attention helps manage the information held in visual working memory (vWM). Perceptual attention selects the stimuli to be represented in vWM, whereas internal attention prioritizes information already in $\mathrm{vWM}$. In the present study we assessed the spatial precision of perceptual and internal attention in vWM. Participants encoded eight colored dots for a local-recognition test. To manipulate attention, a cue indicated the item most likely to be tested ( $\sim 5 \% \%$ validity). The cue appeared either before the onset of the memory array (precue) or during the retention interval (retrocue). The precue guides perceptual attention to gate encoding into vWM, whereas the retrocue guides internal attention to prioritize the cued item within vWM. If attentional selection is spatially imprecise, attention should be preferentially allocated to the cued location, with a gradual drop-off of attention over space to nearby uncued locations. In this case, memory for uncued locations should vary as a function of their distance from the cued location. As compared to a no-cue condition, memory was better for validly cued items but worse for uncued items. The spatial distance between the uncued and cued locations modulated the cuing costs: Items close in space to the cued location were insulated from cuing costs. The extension of this spatial proximity effect was larger for precues than for retrocues, mostly because the benefits of attention were larger for precues. These results point to similar selection principles between perceptual and internal attention and to a critical role of spatial distance in the selection of visual representations.
\end{abstract}

Keywords Spatial attention $\cdot$ Working memory $\cdot$ Distribution of attention $\cdot$ Focus of attention

Attention operates on representations of perceptual inputsalso known as perceptual attention - and on representations sustained only in mind-also known as internal attention (Chun, Golomb, \& Turk-Browne, 2011). Here we are concerned with the operation of perceptual and internal attention on visual working memory (vWM). Perceptual attention controls which perceptual inputs get access to vWM, whereas internal attention prioritizes one over several representations simultaneously held in vWM.

There are many similarities between the effects of perceptual and internal attention on vWM. For example, Griffin and Nobre

Electronic supplementary material The online version of this article (https://doi.org/10.3758/s13423-018-1471-4) contains supplementary material, which is available to authorized users.

Alessandra S. Souza

a.souza@psychologie.uzh.ch

1 Department of Psychology - Cognitive Psychology Unit, University of Zurich, Binzmühlestrasse 14/22, 8050 Zurich, Switzerland
(2003) asked participants to retain four colors in vWM for a single-item recognition test. To manipulate perceptual or internal attention, one memory location was cued as likely to be tested either prior to the onset of the memory array (henceforth, the precue) or during the maintenance phase (the retrocue). When the pre- or retrocue was valid, responses were faster and more accurate than in a baseline condition with a noninformative cue. When the cues were invalid - that is, when an uncued item was tested - costs of cuing were observed in comparison to the baseline (see Souza \& Oberauer, 2016, for a recent review). Furthermore, Nobre and colleagues have uncovered largely overlapping neural networks engaged by precues and retrocues (for reviews, see Gazzaley \& Nobre, 2012; Lepsien \& Nobre, 2006; Myers, Stokes, \& Nobre, 2017), in line with the hypothesis that the two types of cues operate in similar ways to prioritize information.

Here our main goal was to assess the spatial precision of perceptual and internal attentional selection within vWM, as indexed by the precue and retrocue effects, respectively. Imprecise spatial selection entails that attention is preferentially allocated to one location (e.g., the cued one) with a gradual 
fall-off over space to nearby locations. Schmidt, Vogel, Woodman, and Luck (2002) used precues to show that perceptual attention in vWM spills over to nearby uncued locations. In their study, the precued items were remembered with a high probability, and performance in invalid precue trials was better, the closer the tested item was to the cued location, indicating that nearby items were also partially attended to.

This finding begs the question of whether a similar effect occurs for internal attentional selection. This question can be investigated using retrocues: If internal attention is imprecisely allocated to the retrocued location, the cuing costs for invalidly retrocued items should also vary as a function of the spatial distance between the retrocued item and the tested item. Imprecision in the spatial allocation of internal attention has implications for theories about the focus of attention in vWM. According to the embedded-process model (Cowan, 1999), the focus of attention can hold several items simultaneously. An imprecise focus that holds not only the one retrocued item, but its neighbors as well, could be assumed to be advantageous because it extends the beneficial effect of attention to those neighbors. Other theories (McElree, 2006; Oberauer, 2002) assume that the focus of attention serves as a selection device that is functionally constrained to hold a single item. Concurrent selection of multiple items would increase the chance of confusions between items, thereby undermining this selection function (see Oberauer, 2013, for a review). From this theoretical perspective, we should expect that a focus of attention optimally tuned to its task would be confined fairly precisely to a single item. Some accounts of the retrocue effect also assume that the internal focus of attention is constrained to a single item (Myers et al., 2017), which can then directly assume the function of guiding action.

By using partially valid cues to guide attention before encoding (precues) and before retrieval (retrocues), we assessed whether imprecise spatial allocation of perceptual or internal attention, respectively, modulates the costs for tests of uncued items as a function of their spatial distance from the cued location. If this were the case for both precues and retrocues, this would indicate that the focus of attention is not constrained to a single item in either case. In contrast, if this effect occurred only in the precue condition, it would indicate that only perceptual attention is spatially imprecise, but internal attention is not.

\section{Experiment 1}

\section{Method}

\section{Participants}

Sixty students (from 18 to 35 years old) at the University of Zurich took part in two 1-h sessions in exchange for course credit or 30 CHF. The participants were evenly split into two groups, receiving two experimental versions (Exps. 1a and 1b) that differed only regarding the time available to encode the memoranda. For all experiments reported here, the participants read and signed an informed-consent form prior to testing and were debriefed at the end. The experimental protocol was in accordance with the ethical regulations of the Faculty of Arts and Social Sciences of the University of Zurich.

\section{Materials and procedure}

The experiments were programmed using Psychophysics Toolbox 3 (Brainard, 1997), implemented in Matlab. Participants were tested in individual booths, where they sat approximately $50 \mathrm{~cm}$ away from the computer screen (viewing distance was unconstrained).

Participants completed a color recognition task with a cuing manipulation (precue, retrocue, or no cue). In the no-cue condition (see Fig. 1), each trial started with a white fixation cross presented against a gray background $(0.5 \mathrm{~s})$. Next, eight colored dots (radius $0.83^{\circ}$ ) were shown for either $1 \mathrm{~s}$ (Exp. 1a) or $0.1 \mathrm{~s}$ (as had been used by Schmidt et al., 2002; Exp. 1b). The memoranda were randomly sampled from a set of 12 colors (beige, yellow, light green, dark green, light blue, dark blue, purple, magenta, brown, red, orange, and black). The dots were evenly spaced along an invisible circle (radius $5^{\circ}$ ) centered on the middle of the screen. A blank screen was shown after array offset ( $1 \mathrm{~s}$; the retention interval), followed by the presentation of a test stimulus in one of the memory locations until a response was given. Participants judged whether the test stimulus had the same color as the memory item that had appeared in that location: They pressed the left or the right arrow key to indicate a positive or negative response, respectively. A positive (matching) test stimulus was shown in 50\% of the trials. The negative test stimuli were of two types: a color not presented in the memory array (new probe, $25 \%$ of the trials) or a color that had been shown at another position than the tested one (intrusion probe). After the response, visual feedback regarding the correctness of the response was presented for $0.5 \mathrm{~s}$. The next trial started after a 1.5 -s blank interval.

Precue trials only differed from no-cue trials in that the fixation cross was followed by the presentation (for $0.1 \mathrm{~s}$ ) of a white arrow pointing to the location of one of the upcoming memory items, and the memory array was shown $1 \mathrm{~s}$ thereafter. Retrocue trials, in contrast, only differed from no-cue trials regarding the events unfolding before the test: After the 1-s retention interval, a white arrow $(0.1 \mathrm{~s})$ pointed to one of the memory locations, and the test stimulus was shown $1 \mathrm{~s}$ thereafter. The 1-s postcue interval in both cuing conditions was included in order to provide ample time for attention to be focused on the cued location. The cues were valid in $65 \%$ of the trials. In the remaining trials, one of the uncued items was tested. The distance between the cued (hereafter, D0) and 


\section{No-Cue Condition}

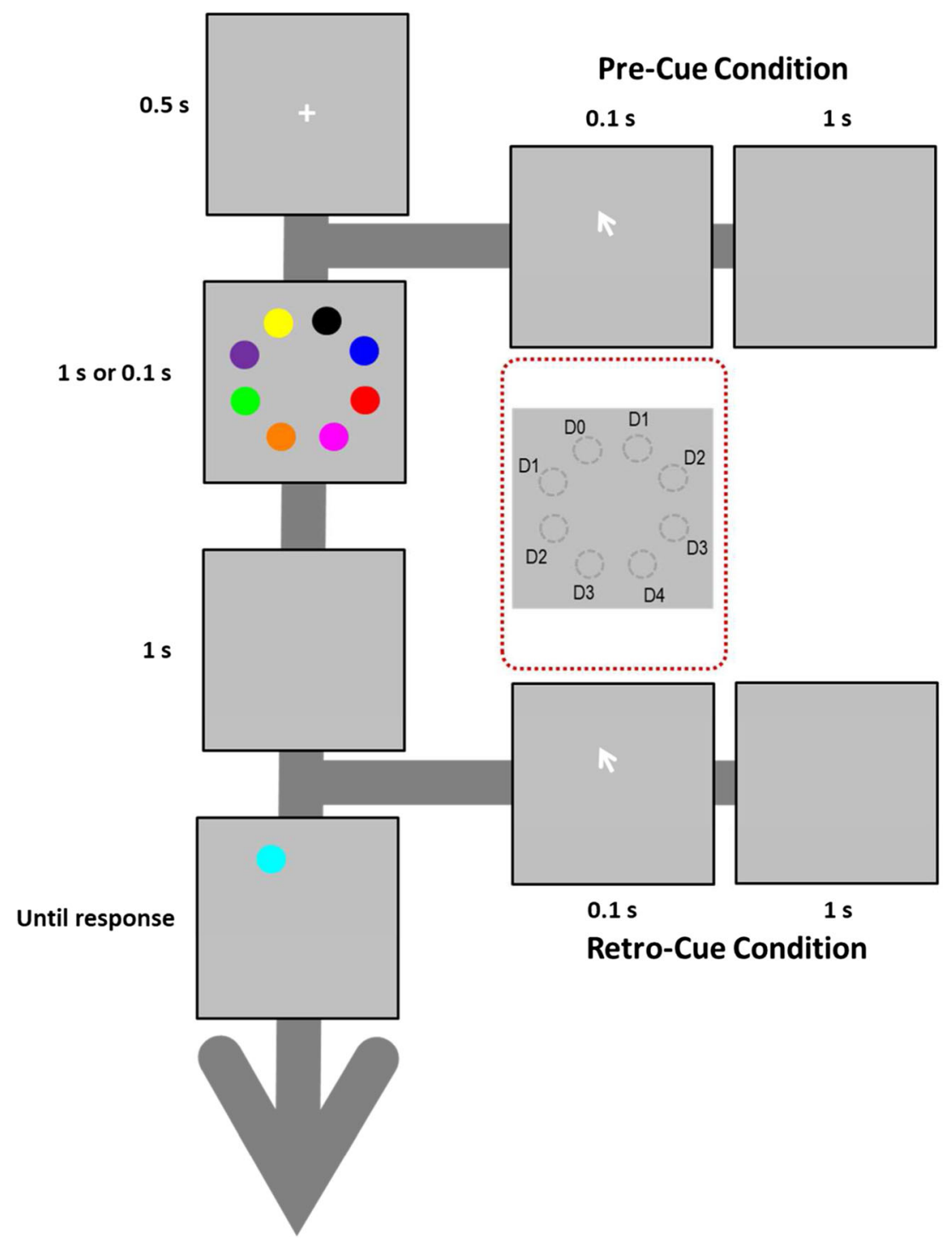

Fig. 1 Illustration of the flow of events in no-cue trials. Precue and retrocue trials only differed from no-cue trials in terms of the presentation of the cue and of the blank interval before encoding or

uncued items was varied in four steps (D1-D4), as is indicated in the inset in Fig. 1, with D1 being the closest location to the cued one ( $45^{\circ}$ away), and $\mathrm{D} 4$ being the location $180^{\circ}$ away.

Across the two experimental sessions, participants completed a total of 800 trials: 320 precue trials, 320 retrocue trials, and 160 no-cue trials. Among each set of 320 cued trials, 208 were valid trials and 112 were invalid ones. In invalid-cue trials, the items at the distances 1-4 were equally likely to be tested (yielding 28 trials per distance). At the beginning of the experiment, participants were instructed about the three trial types (no cue, precue, and retrocue) and told that the cues would indicate the test item in the majority of the trials. They were further instructed to repeat continuously aloud "der-die-das" in order to prevent the use of verbal memory.

before the memory test, respectively. The inset within the dotted line illustrates the spatial distance between the cued item (D0) and the remaining memory items (D1-D4, in steps of $45^{\circ}$ ) in both cue conditions

\section{Data analysis}

We submitted our data to a Bayesian analysis of variance (ANOVA; Rouder, Morey, Speckman, \& Province, 2012) using the BayesFactor package (Morey, Rouder, \& Jamil, 2014) for R (R Core Team, 2014). A Bayesian ANOVA computes the strength of the evidence in the data in favor of including or omitting an effect of interest. The relative evidence for one model over another is the Bayes factor (BF). In the present article, BFs above 1 indicate that the data are more likely under the alternative hypothesis, whereas BFs below 1 indicate that the data are more likely under the null hypothesis. It is common to consider BFs between 0.33 and 3 as weak evidence in favor of a 
hypothesis, whereas BFs below 0.10 or above 10 are considered strong support for the null or the alternative hypothesis, respectively.

In addition, we analyzed the data using a hierarchical Bayesian logistic regression model, which has the advantage of using the accuracy of each trial as the dependent variable, as opposed to the proportion of correct responses aggregated within each design cell. The same pattern of results was obtained (see the online supplementary material).

The data and analysis scripts for all experiments reported here and in the supplementary material are available at the Open Science Framework: https://osf.io/vz89r/.

\section{Results}

Figure $2 \mathrm{~A}$ shows the proportions of correct responses as a function of cue condition and spatial distance between the cued and tested items, separately for each experimental version.

\section{Valid-cue benefits}

To assess valid-cue benefits, we entered in two separate ANOVAs the factors cue condition (no cue vs. valid precue, or no cue vs. valid retrocue) and experimental version (1a vs. 1b), which involved only a difference in encoding time. Valid precues improved accuracy as compared to no-cue trials $\left(\mathrm{BF}_{10}=1.9 \times 10^{27}\right)$. Experimental version had no effect $\left(\mathrm{BF}_{10}=0.22\right)$; however, there was weak evidence for a valid Precue $\times$ Experiment interaction $\left(\mathrm{BF}_{10}=2.55\right)$, due to the somewhat larger validprecue benefit in Experiment $1 \mathrm{~b}$. Likewise, valid retrocues improved accuracy $\left(\mathrm{BF}_{10}=1.4 \times 10^{9}\right)$. There was weak evidence against a main effect of experiment $\left(\mathrm{BF}_{10}=0.55\right)$, and against a valid Retrocue $\times$ Experiment interaction $\left(\mathrm{BF}_{10}=0.44\right)$. Finally, we compared the sizes of the cuing benefits with a $t$ test, which provided overwhelming support $\left(\mathrm{BF}_{10}=3.66 \times 10^{17}\right)$ for a larger precue benefit than retrocue benefit.

\section{Invalid-cue costs}

To assess invalid cuing costs (across all distances), we conducted two ANOVAs, entering cue condition (no cue vs. invalid precue, or no cue vs. invalid retrocue) and experiment as factors. We found evidence for an invalid-precue cost $\left(\mathrm{BF}_{10}=6.77\right)$ and an effect of experiment $\left(\mathrm{BF}_{10}=\right.$ 3.05 ), as well as weak evidence against their interaction $\left(\mathrm{BF}_{10}=0.56\right)$. The effect of experiment reflects the somewhat lower levels of performance in Experiment 1b (with shorter encoding time). There was very strong evidence for invalid-retrocue costs $\left(\mathrm{BF}_{10}=46,370\right)$, ambiguous evidence for a main effect of experiment $\left(\mathrm{BF}_{10}=1.49\right)$, but no interaction between them $\left(\mathrm{BF}_{10}=0.28\right)$. Overall, the comparison of the sizes of the cuing costs between cue types showed support for smaller costs with precues than with retrocues $\left(\mathrm{BF}_{10}=10\right)$.

\section{Spatial-distance effect}

Our main question regarded any modulation of invalidcuing effects by the spatial distance between the cued location and the uncued but tested location, and whether this effect differed depending on cue type. For this analysis, we took only invalidly cued trials and assessed the effects of spatial distance, cue condition (precue vs. retrocue), experiment, and their interaction. The best model $\left(\mathrm{BF}_{10}=\right.$ $1,736.5)$ included the effects of spatial distance, cue condition, and experiment, as well as the Distance $\times$ Cue interaction. Critically, inclusion of the Distance $\times$ Cue interaction was supported by $\mathrm{BF}_{10}=4.71$, reflecting the fact that the cuing costs only varied with spatial distance in the precue condition. To get to the bottom of this interaction, we assessed the evidence for a spatial-distance effect in each cue condition separately. There was strong evidence against an effect of spatial distance in the retrocue condition $\left(\mathrm{BF}_{10}=\right.$ 0.04). Conversely, in the precue condition this effect was strongly supported $\left(\mathrm{BF}_{10}=970\right)$.

Figure $2 \mathrm{~A}$ suggests that the modulation of cuing costs by distance is mainly due to items at $\mathrm{D} 1$ ( $45^{\circ}$ away) being insulated from cuing costs in the precue condition. When considering only this distance, a $t$ test showed no evidence for a precue cost $\left(\mathrm{BF}_{10}=0.09\right)$, but overwhelming support for a retrocue cost $\left(\mathrm{BF}_{10}=3,940\right)$.

\section{Discussion}

Experiment 1 showed that objects in the vicinity of the cued location were insulated from cuing costs in the precue but not in the retrocue condition, suggesting that perceptual and internal attention differ in their spatial imprecision. There is one caveat, though: The precue effect was much larger than the retrocue effect. It is, therefore, possible that the observed interaction reflects a scaling artifact. To address this issue, we modeled the data of the two cue conditions with a hierarchical Bayesian exponential model assuming that the accuracy (in logit scale) decreased exponentially with the cued-tested spatial distance (0 to 3$).{ }^{1}$ The exponential is described by three parameters: (1) the asymptote, which here reflects the cuing costs at far locations; (2) the intercept, reflecting the strength of the cuing effect at distance 0 ; and (3) the rate of change as a

\footnotetext{
${ }^{1}$ We excluded distance $4\left(180^{\circ}\right)$ because the tendency toward an increase in accuracy in the precue condition at this distance could distort the fitting of the exponential model.
} 
a

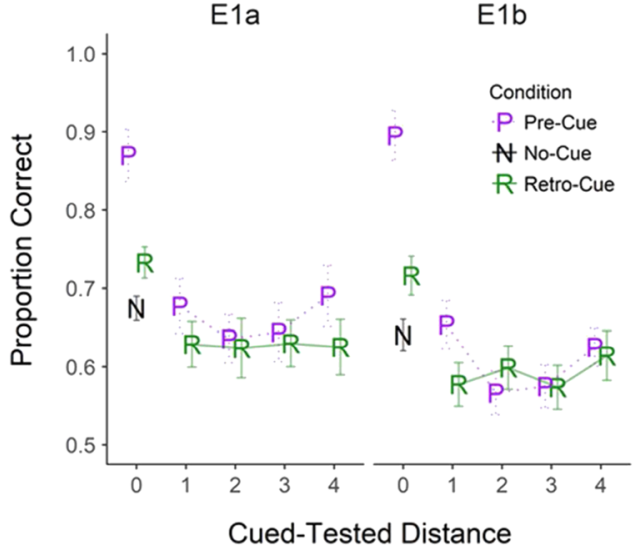

Fig. 2 Proportions of correct responses in each experimental condition as a function of the spatial distance ( $\mathrm{bin}=45^{\circ}$ ) between the cued and the tested item location. (A) Data from Experiments 1a and 1b (E1a, E1b; min. distance $\left.=45^{\circ}\right)$. (B) Data from Experiment $2(\mathrm{E} 2$; min. distance $=$

function of spatial distance. The general form of our model was as follows:

$$
\begin{aligned}
\operatorname{Logit}\left(\operatorname{accuracy}_{\mathrm{t}, \mathrm{p}, \mathrm{c}, \mathrm{e}}\right)= & \text { Asymptote }_{\mathrm{p}, \mathrm{e}}+\text { Intercept }_{\mathrm{p}, \mathrm{c}, \mathrm{e}} \\
& \times \exp \left(- \text {-Rate }_{\mathrm{c}} \times \text { Distance }\right)
\end{aligned}
$$

with $\mathrm{t}$ standing for the trial, $\mathrm{p}$ for participant, $\mathrm{c}$ for cue, and e for experiment. Critically, we built two models that differed only regarding whether we included an effect of cue condition on the rate parameter (two-rate model) or did not (one-rate model; see the model and results in the https://osf.io/vz89r/).

The two-rate model estimated a slightly higher rate of decrease in accuracy over distance for the retrocue than for the precue condition, but this difference was not credibly different from zero $\left(\mathrm{BF}_{10}=0.70\right.$; see Fig. 3). We also compared the fit of the one-rate versus the two-rate model using the deviance information criterion (DIC), a metric for comparing hierarchical Bayesian models. The one-rate model had a slightly lower DIC $(2,560.6)$ than the two-rate model $(2,566.8)$, which is indicative of a better fit. Hence, all in all, the data of Experiment 1 seem ambiguous regarding a difference in the rate of spatial imprecision across cue conditions.

\section{Experiment 2}

The exponential modeling of Experiment 1 provided weak evidence against an effect of cue type on the rate of spatial imprecision. The predictions in Fig. 3A suggest, however, that we might be able to clarify whether the two cue conditions differ in rate by obtaining a more fine-grained measure of the spatial gradient. Hence, in Experiment 2 we doubled the number of possible spatial locations for the memoranda, thereby

\section{b}

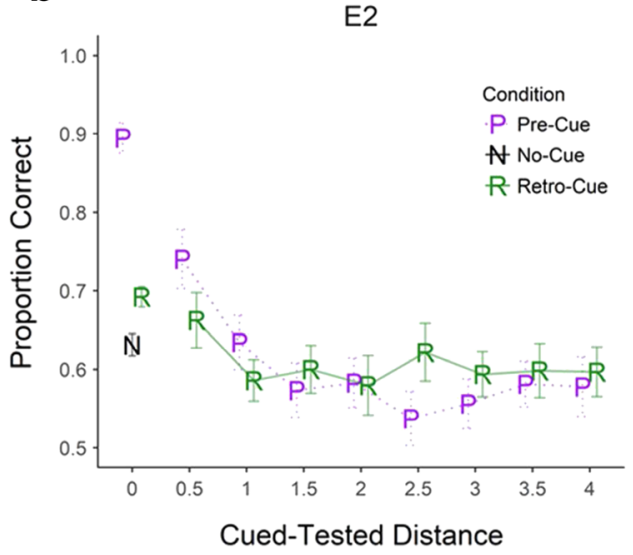

$22.5^{\circ}$ ). In the no-cue condition, no cue was presented before the test; hence, we classified it as having a distance of 0 . Error bars depict $95 \%$ within-subjects confidence intervals (Morey, 2008)

reducing the size of the smallest separation between items to $22.5^{\circ}$ (instead of $45^{\circ}$ ). By doing so, we increased the resolution of our measurement of the spatial gradient. If the allocation of attention in the retrocue is also spatially imprecise, we should observe that the items at the closest location to the cued one are now spared from cuing costs, akin to the observation for precues.

\section{Method}

\section{Participants and procedure}

Forty students took part in Experiment 2. The procedure was the same as in Experiment 1b, with three exceptions. First, we increased the number of possible memory locations from eight to 16 , thereby reducing the minimal distance between two items to $22.5^{\circ}$. Second, for every trial we randomly selected half of the locations to be occupied by memory items. Third, the cues were valid in $60 \%$ of the trials (192 of 320 trials), and the invalid-cue trials were evenly distributed across the eight spatial distances $\left(22.5^{\circ}\right.$ to $180^{\circ}$ in steps of $\left.22.5^{\circ}\right)$, yielding 16 trials per distance.

\section{Results}

Figure 2B shows accuracy as a function of spatial distance in Experiment 2. For better comparability across experiments, we also binned distances in steps of $45^{\circ}$. Our main interest was to observe the cuing costs for neighboring items (D0.5 and D1). There was strong evidence against cuing costs at the smallest spatial distance $\left(\mathrm{D} 0.5: 22.5^{\circ}\right)$ in both the precue $\left(\mathrm{BF}_{10}=0.02\right)$ and retrocue $\left(\mathrm{BF}_{10}=0.07\right)$ conditions. At a distance of $45^{\circ}$ (D1), only the precue condition showed evidence against cuing costs $\left(\mathrm{BF}_{10}=0.15\right)$, whereas the evidence for a retrocue cost was strong $\left(\mathrm{BF}_{10}=28.8\right)$, replicating the results of Experiment 


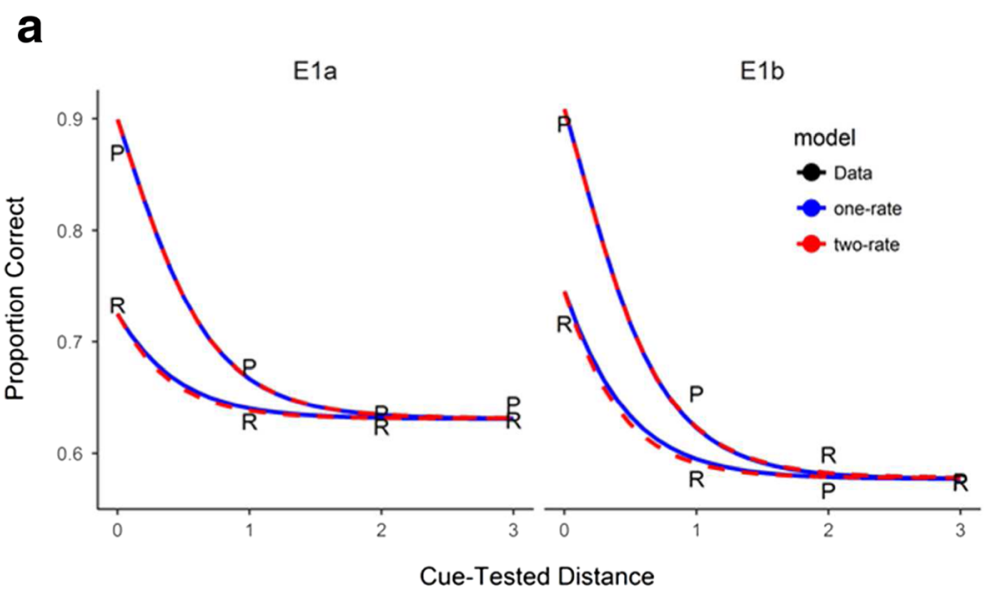

b

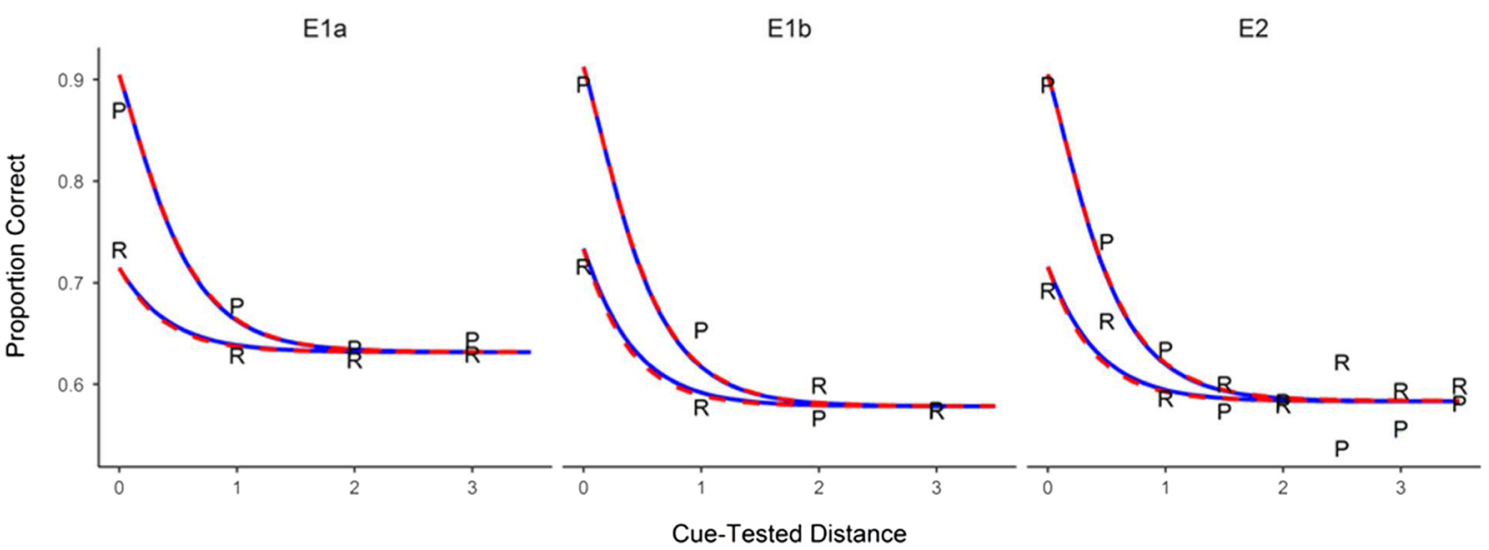

Fig. 3 Predictions of the exponential models (lines) plotted alongside the data (letters: P for precue, $\mathrm{R}$ for retrocue) in each experiment. (A) Predictions of the models fitted simultaneously to the data of

1. Modeling the data of the three experiments together (see Fig. 3B) with the exponential function showed evidence against a difference in the rate parameter across cue conditions $\left(\mathrm{BF}_{10}=\right.$ $0.36)$, and the one-rate model had a better fit $(\mathrm{DIC}=5,375.8)$ than the two-rate model ( $\mathrm{DIC}=5,388.6$ ).

\section{Discussion}

Experiment 2 showed that the spatial imprecisions of the two cue conditions, when measured as the rate of an exponential drop-off of accuracy, were similar. When the minimum distance between items was reduced, we were able to measure a spread of the retrocue effect to nearby locations. These results indicate that the two cue conditions differ mainly in the strength of the cuing effect, not in their selectivity over space.

\section{General discussion}

We guided perceptual attention to gate encoding into vWM by using precues, and we guided internal attention to modulate retrieval from vWM by using retrocues. In both cases, the cues
Experiments $1 \mathrm{a}$ and $1 \mathrm{~b}$. (B) Predictions of the model fitted simultaneously to the data of Experiments 1a, 1b, and 2

were only partially valid, such that in some proportion of trials, uncued items were also tested. Our main aim was to assess whether performance for uncued items would vary as a function of their spatial distance from the cued location. If attentional allocation is spatially imprecise, then items in the vicinity of the cued location would also be partially attended to, and memory for these items should be better than for items farther away. Previous research has shown that this is the case for the allocation of perceptual attention in order to gate encoding into vWM (Schmidt et al., 2002). Here we addressed the question of whether internal attentional selection from vWM is also imprecise, such that when participants attempt to select one vWM content, they also partially select other items at nearby locations. Experiment 1 initially pointed to differences in the ways that spatial attention was allocated to the cued locations: The retrocue effect did not spill over to nearby locations, whereas the precue effect did. Modeling of the data, however, pointed to the possibility that measurement of the rate of spatial imprecision in the proportion-correct scale was confounded by the strength of the attentional benefit. To bypass this limitation, we reduced the spatial distance between items by half $\left(22.5^{\circ}\right.$, Exp. 2), which then allowed us to also observe a neighbor- 
sparing effect in the retrocue condition. These results indicate that the metric distance between items (separation in space) rather than ordinal separation (neighbors vs. nonneighbors) is critical in the spatial allocation of attention in both domains.

One may wonder whether the appearance of a steeper spatial gradient for retrocues in Experiment 1 could be minimized under a lower memory load. To answer this question, we ran an additional experiment (see the online supplementary material) in which we varied memory load (two, four, or eight items; minimum spatial distance of $45^{\circ}$ ) and the presence of a retrocue versus of no cue. There were clear memory load effects, valid-retrocue benefits, and invalid-retrocue costs. In line with Experiment 1, we found no evidence supporting a spillover of internal attention to locations $45^{\circ}$ away on the circle for any level of memory load.

Many studies have pointed to similarities in the ways in which perceptual and internal attention operate on vWM representations (Gazzaley \& Nobre, 2012; Griffin \& Nobre, 2003; Sahan, Verguts, Boehler, Pourtois, \& Fias, 2016). Some other studies, however, have pointed to dissociations. For example, Makovski and Jiang (2007) showed that spatial cuing of multiple items simultaneously was beneficial in the case of precues, but not of retrocues, and Tanoue and Berryhill (2012) showed that the allocation of perceptual attention through precues depends on the eccentricity of the objects, but that this was not the case for retrocues. With regard to the precision of spatial allocation, our data suggest that both perceptual and internal attention spread along the same spatial gradient. Given that the benefits of internal attention are smaller than those of perceptual attention, the distance over which the cuing effect spreads in the retrocue condition is, however, reduced.

Our results indicate that the selection of representations in vWM is not tightly constrained to one item's spatial location. One interpretation of this result is that the focus of attention in working memory sometimes selects two spatially close items simultaneously. This would raise their chance of being encoded (when precued) or retrieved (when retrocued), but at the same time might raise their chance of being confused with each other. Other studies (Bays, 2016; Emrich \& Ferber, 2012; Oberauer \& Lin, 2017; Rerko, Oberauer, \& Lin, 2014; Sahan et al., 2016) have shown that the degree of spatial overlap between items increases the likelihood of these items being confused with each other (binding error). Attending to them simultaneously would raise the accessibility of both, thereby raising the chance of confusing the target with its close neighbor at test - this would diminish the cuing benefit. An alternative interpretation of the present findings is that, on each trial, only one item is selected by attention, and the spatial gradient reflects the probability of misselection: On some trials, participants attend not to the cued item but to its close neighbor. In this scenario, only one item is attended at any time, and the problem of an increased chance of confusion does not arise.
Author's Note Alessandra S. Souza, Mirko Thalmann, \& Klaus Oberauer, Department of Psychology, University of Zurich. We thank Laura Rerko for her contribution in an earlier phase of this project. This project was supported by a grant from the Forschungskredit to A.S. Souza (project FK-13-083) and grants from the Swiss National Science Foundation to M. Thalmann (project 168257) and K. Oberauer (project 143333).

\section{References}

Bays, P. M. (2016). Evaluating and excluding swap errors in analogue tests of working memory. Scientific Reports, 6, 19203. doi:https:// doi.org/10.1038/srep19203

Brainard, D. H. (1997). The psychophysics toolbox. Spatial Vision, 10, 433-436. doi:https://doi.org/10.1163/156856897X00357

Chun, M. M., Golomb, J. D., \& Turk-Browne, N. B. (2011). A taxonomy of external and internal attention. Annual Review of Psychology, 62, 73-101. doi:https://doi.org/10.1146/annurev.psych.093008.100427

Cowan, N. (1999). An embedded-process model of working memory. In A. Miyake \& P. Shah (Eds.), Models of working memory: Mechanisms of active maintenance and executive control (pp. 62101). Cambridge, UK: Cambridge University Press.

Emrich, S. M., \& Ferber, S. (2012). Competition increases binding errors in visual working memory. Journal of Vision, 12(4), 12. doi:https:// doi.org/10.1167/12.4.12

Gazzaley, A., \& Nobre, A. C. (2012). Top-down modulation: Bridging selective attention and working memory. Trends in Cognitive Sciences, 16, 129-135. doi:https://doi.org/10.1016/j.tics.2011.11. 014

Griffin, I. C., \& Nobre, A. C. (2003). Orienting attention to locations in internal representations. Journal of Cognitive Neuroscience, 15, 1176-1194. doi:https://doi.org/10.1162/089892903322598139

Lepsien, J., \& Nobre, A. C. (2006). Cognitive control of attention in the human brain: Insights from orienting attention to mental representations. Brain Research, 1105, 20-31. doi:https://doi.org/10.1016/j. brainres.2006.03.033

Makovski, T., \& Jiang, Y. V. (2007). Distributing versus focusing attention in visual short-term memory. Psychonomic Bulletin \& Review, 14, 1072-1078. doi:https://doi.org/10.3758/BF03193093

McElree, B. (2006). Accessing recent events. In B. H. Ross (Ed.), The psychology of learning and motivation (Vol. 46, pp. 155-200). San Diego, CA: Academic Press. doi:https://doi.org/10.1016/S00797421(06)46005-9

Morey, R. D. (2008). Confidence intervals from normalized data: A correction to Cousineau (2005). Tutorials in Quantitative Methods for Psychology, 4, 61-64.

Morey, R. D., Rouder, J. N., \& Jamil, T. (2014). BayesFactor: Computation of Bayes factors for common designs ( $\mathrm{R}$ package version 0.9.9). Retrieved from http:/CRAN.R-project.org/package= BayesFactor

Myers, N. E., Stokes, M. G., \& Nobre, A. C. (2017). Prioritizing information during working memory: Beyond sustained internal attention. Trends in Cognitive Sciences, 21, 449-461. doi:https://doi.org/ 10.1016/j.tics.2017.03.010

Oberauer, K. (2002). Access to information in working memory: Exploring the focus of attention. Journal of Experimental Psychology: Learning, Memory, \& Cognition, 28, 411-421. doi: https://doi.org/10.1037/0278-7393.28.3.411 
Oberauer, K. (2013). The focus of attention in working memory: From metaphors to mechanisms. Frontiers in Human Neuroscience, 7 , 673. doi:https://doi.org/10.3389/fnhum.2013.00673

Oberauer, K., \& Lin, H.-Y. (2017). An interference model of visual working memory. Psychological Review, 124, 21-59.

R Core Team (2014). R: A language and environment for statistical computing. Vienna, Austria: R Foundation for Statistical Computing. Retrieved from www.R-project.org

Rerko, L., Oberauer, K., \& Lin, H.-Y. (2014). Spatial transposition gradients in visual working memory. Quarterly Journal of Experimental Psychology, 67, 3-15.

Rouder, J. N., Morey, R. D., Speckman, P. L., \& Province, J. M. (2012). Default Bayes factors for ANOVA designs. Journal of Mathematical Psychology, 56, 356-374. doi:https://doi.org/10. 1016/j.jmp.2012.08.001
Sahan, M. I., Verguts, T., Boehler, C. N., Pourtois, G., \& Fias, W. (2016). Paying attention to working memory: Similarities in the spatial distribution of attention in mental and physical space. Psychonomic Bulletin \& Review, 23, 1190-1197.

Schmidt, B. K., Vogel, E. K., Woodman, G. F., \& Luck, S. J. (2002). Voluntary and automatic attentional control of visual working memory. Perception \& Psychophysics, 64, 754-763. doi:https://doi.org/ 10.3758/BF03194742

Souza, A. S., \& Oberauer, K. (2016). In search of the focus of attention in working memory: 13 years of the retro-cue effect. Attention, Perception, \& Psychophysics, 78, 1839-1860.

Tanoue, R. T., \& Berryhill, M. E. (2012). The mental wormhole: Internal attention shifts without regard for distance. Attention, Perception, \& Psychophysics, 74, 1199-1215. doi:https://doi.org/10.3758/s13414012-0305-0 